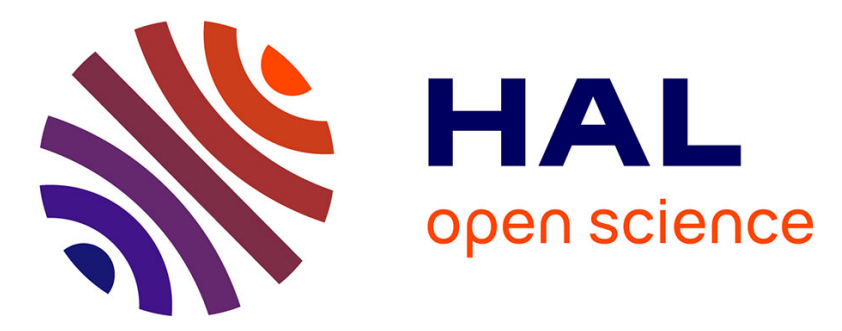

\title{
Optimal packet allocation methods in multi-beam satellite architecture using FTDMA on the Return Link
}

Dimitri Serrano-Velarde, Emmanuel Lance, Georges Rodriguez-Guisantes, Hector Fenech

\section{- To cite this version:}

Dimitri Serrano-Velarde, Emmanuel Lance, Georges Rodriguez-Guisantes, Hector Fenech. Optimal packet allocation methods in multi-beam satellite architecture using FTDMA on the Return Link. 29th AIAA International Communications Satellite Systems Conference (ICSSC-2011), Nov 2011, Nara, Japan. hal-00732461

\section{HAL Id: hal-00732461 https://hal.science/hal-00732461}

Submitted on 14 Sep 2012

HAL is a multi-disciplinary open access archive for the deposit and dissemination of scientific research documents, whether they are published or not. The documents may come from teaching and research institutions in France or abroad, or from public or private research centers.
L'archive ouverte pluridisciplinaire HAL, est destinée au dépôt et à la diffusion de documents scientifiques de niveau recherche, publiés ou non, émanant des établissements d'enseignement et de recherche français ou étrangers, des laboratoires publics ou privés. 


\title{
Optimal packet allocation methods in multi-beam satellite architecture using FTDMA on the Return Link
}

\author{
Dimitri Serrano-Velarde ${ }^{1}$, Dr. Emmanuel Lance ${ }^{2}$ and Dr. Hector Fenech ${ }^{3}$ \\ Eutelsat SA, Paris, France, 75502 \\ and \\ Dr. Georges Rodriguez-Guisantes ${ }^{4}$ \\ Telecom ParisTech, Paris, France, 75634
}

This paper introduces new techniques to enhance the construction of frames in a MFTDMA communication system for a high throughput broadband Satellite system. It is a function of one main parameter: the Carrier-over-Interference (C/I) performance at antenna level taking into account geographical constraints. The satellite architecture uses a multi-beam coverage implementing frequency re-use and spatial separation on a Return Link.

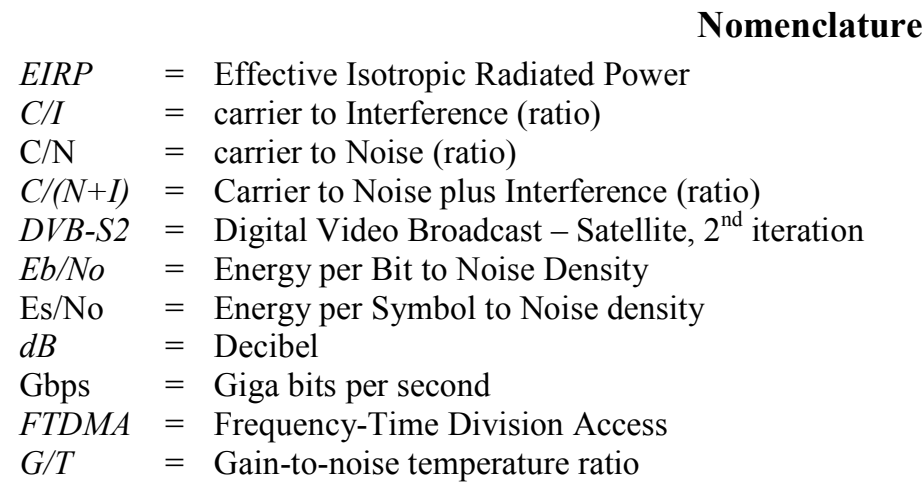

\section{Introduction}

$\mathrm{M}$ ULTI-BEAM satellite coverages are being used more and more widely by satellite operators like Eutelsat with KaSat or Viasat with ViaSat1. Those coverages allow an operator to gain more flexibility and capacity by using its resources more efficiently on a wide coverage thanks to frequency re-use combined with spatial separation, in the shape of FTDMA encoding.

Consequently, the way of building the data frames is a potential bottleneck for the Return Link of a broadband communication system. Depending on the data packet association, a system can be optimized according to a certain performance parameter such as the Carrier-to-Interference (C/I) performance at antenna level.

$1 \mathrm{PhD}$ Student and Communication Engineer, Department of Missions and Programmes, 70, rue balard/dserrano@eutelsat.com.

2 Senior Communication Engineer, Future Department of Missions and Programmes, 70, rue Balard/elance@eutelsat.com.

3 Head of Future Satellite Systems Group, Department of Missions and Programmes, 70, rue Balard/hfenech@eutelsat.com.

4 Research Professor, Département Communications et Electronique, 46 rue Barrault/rodriguez@telecomparistech.fr.

1

American Institute of Aeronautics and Astronautics 
In order to present the scope of the optimization scheme and of the two techniques, we are going first to expose the problematic then the optimization techniques associated with simulation results and the conclusion drawn from these results.

\section{Problem Description}

Satellites have long been used for services related to broadcasting like TV or radio services but the new generation of satellites aims at providing broadband services as a natural extension to the broadcast services ${ }^{1}$. The architecture used for those services is a multi-beam coverage composed of a given number of beams and cell structure, which once put together, generate the whole service area.

One of the specificities of such coverages is the flexibility in terms of on board resource management. It is possible to distribute for instance the power given certain requirements or re-use as much as possible a limited bandwidth (through frequency re-use and spatial separation). However, the major drawback of such architectures is the inter-spot interferences. Those interferences, if poorly designed, can harm any communication.

The traffic being a web traffic is consequently composed of data packets that the satellite has to handle properly as they are transmitted and received via FTDMA frames on the Return link based on very specific protocols.

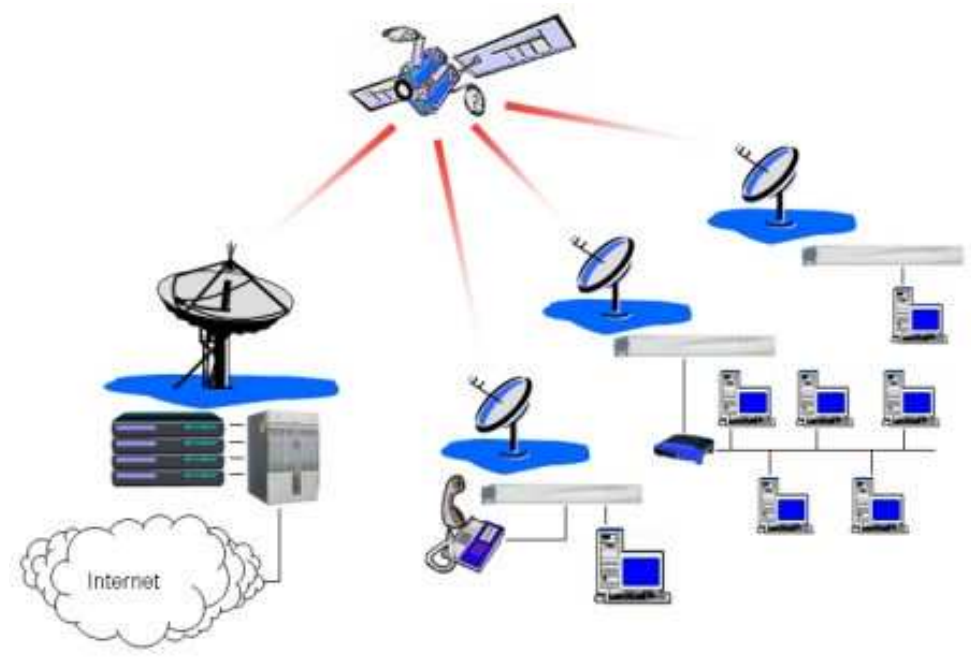

Figure 1: Satellite Communication Scenario

The way of putting together the data packets, in other words the users to be transmitted to the satellite in a FTDMA scheme, can play an important role on the interferences generated at antenna level between each spot. As seen in various publications ${ }^{2,3,4}$ the optimization idea is to influence the cell definion or cell hierarchy. By using the publications $^{5,6}$, it is possible to build up a good foundation for a link budget model and incorporate a precise C/I model.

The problematic is consequently, how to organize the data packets in the current frame on a Return Link before sending it given a performance parameter and taking into account an optimization method based on a distance criteria between each interferer and also the upcoming frames.

The methods we are going to present, aim at optimizing the interferences generated between each spot and consequently the $\mathrm{C} / \mathrm{I}$ which influences heavily the global link budget performances.

The two techniques under test for the optimization of the frames are: a technique based on the beam centers called "Beam centers" and another one based on the user itself called "User centered". Also the system is supposed to be able to predict future traffic over three upcoming frames.

\section{The Antenna and C/I Model}

In order to start the reasoning, it is necessary to generate the antenna system with software tools and convert the radiation diagrams into usable formats for the link budget, in other words EIRP density or G/T matrices. 
The adopted reflector model is designed by taking into account the illumination taper and spillover losses but also by neglecting the scan aberration. It is based on the model proposed by Peter Balling ${ }^{7}$.

First, the aperture distribution from a single feed is calculated by:

$$
g(r)=p+(1-p) *\left(1-\left(\frac{r}{a}\right)^{2}\right)^{n}
$$

with:

- $a$, the radius of the aperture

- $\quad r$, radius in spherical coordinate system

- $p$, the relative edge illumination

- $\quad n$ is typically 1 , but may be changed in the illumination exponent field

The beams are approximated by a linear combination of Bessel functions:

$$
F_{j}(\theta, \phi)=k * a *\left(c_{1}^{*} B F\left(1, k * a x_{j}\right)+c_{2} * B F\left(n+1, k a x_{j}\right)\right)
$$

with:

- $k$, the propagation constant $\frac{2 \pi}{\lambda}$

- $\quad \theta, \phi$, coordinates in a spherical reference system

- $x_{j}$, the distance from beam center, $x_{j}=\sqrt{\left(\left(u-u_{j}\right)^{2}+\left(v-v_{j}\right)^{2}\right)}$

- $\operatorname{BF}(n, x)$, Bessel functions given by:

$$
\left\{\begin{array}{l}
B F(n, x)=2^{n} * n ! * \frac{J_{n}(x)}{x^{n}} \\
B F(n, 0)=1
\end{array}\right.
$$

- $\quad$ The coefficients $c_{1}$ and $c_{2}$ depend on the edge taper $\mathrm{p}$, and are normalized so that $F_{j}{ }^{2}$ yields directivity. Balling uses an analytic approximation to determine directivity. We prefer perform a PO integration of an on-focus feed to determine the directivity, which is more accurate.

Afterwards, based on a cell structure, it is possible to calculate the $\mathrm{C} / \mathrm{I}$ ratio as a performance indicator at antenna level. Indeed, for a multi-beam antenna system implementing frequency reuse and spatial separation, it is important to calculate the interferences generated by all the beams on each other, as the more spots one stacks, the more interference is generated. In a general way, it is possible to define the calculation of the $\mathrm{C} / \mathrm{I}$ as follows:

Let's consider a certain spot $\mathrm{k}$ with a capacity of $C_{k}$. Considering, that the architecture is a mutli-beam coverage with frequency reuse, there will be two type of interferers. Depending on the side of the transmission, there will be the interferers in co-polarization, which are at the same frequency and the same polarization but used in different spatial spots. Their contribution denoted as $I_{c o}$, can be quantified as:

$$
I_{c o}=\sum_{q=0}^{N}\left(D_{q}^{C o}(x)\right)
$$

with:

- $q$, the identifier for an interferring spot

- $\quad N$, the total number of interferers in Co-polarization

- $x$, the point defined by a coordinate system

- $D_{q}^{C o}$, the directivity in Co-polarization for the interferer $\mathrm{q}$

There are also the interferers related to the cross polarization, which are the spots at the same frequency but with a directly opposite polarization. Their contribution denoted as $I_{c x}$ is as follows: 
with:

$$
I_{c x}=\sum_{p=0}^{M}\left(D_{P}^{C x}(x)\right)
$$

- $\quad p$, the identifier for an interferring spot

- $M$, the total number of interferers in the orthogonal polarization

- $\quad x$, the point defined by a coordinate system

- $D_{p}^{C x}$, the directivity in the orthogonal polarization of interfering spot $\mathrm{p}$

Combined all together, the $\mathrm{C} / \mathrm{I}_{\text {tot }}$ calculation is done as follows:

$$
\frac{C}{I_{t o t}}=\frac{C_{k}}{I_{c o}+I_{c x}}
$$

It is also important to include the Beam Pointing Error (BPE), which can influence heavily on the performances.

\section{The "Beam Centers" Technique}

The first method of optimization will be based on the center of the beams of each spot. Indeed, the idea is to identify the weakest user of any spot in terms of capacity and see if a C/I criteria is verified. If not, one takes the center of the beam of this weakest user and then looks for all the different users who are emitting at the same time and interfering causing a low C/I. Once, the closest interfering user is identified, he is swapped with another one from the same spot but from a different frame emitted later in the queue. The interference is consequently delayed. However, the packet can't be delayed more than three times as the user who is waiting for this packet needs to receive it.

$$
L_{C I}=\min \left(\left(\frac{C}{I}\right)_{\text {users }}\right)
$$

with:

- $\quad L_{C I}$, the lowest $\mathrm{C} / \mathrm{I}$ value

Through the weakest $\mathrm{C} / \mathrm{I}$, it is possible to identify the weakest user and the spot covering it. Then the closest interferer is identified through the distance towards the beam center:

$$
D_{I}=\min \left(x_{\text {Intf }}-x_{\text {weakestBeam }}\right)
$$

With:

- $D_{I}$, corresponding to the smallest distance

- $x_{\text {Intf }}$, corresponds to the coordinates of the interferers

- $x_{\text {weakestBeam }}$, corresponds to the coordinates of the Beam containing the weakest user

The closest interferer is swapped with another one of the same spot but which is farther away from the weakest user.

\section{The User Centered technique}

The second method is based on a combination with a distance criterion with regard to the user itself. Indeed, instead of looking for the closest interferer in comparison to the beam center, this method is going to look for the closest user towards the weakest user. As above it will replace the closest interferer with another one from the same spot but further away.

$$
L_{C I}=\min \left(\left(\frac{C}{I}\right)_{\text {users }}\right)
$$

with:

- $\quad L_{C I}$, the lowest $\mathrm{C} / \mathrm{I}$ value 
Through the weakest C/I, it is possible to identify the weakest user and the spot covering it. Then the closest interferer is identified through the distance towards the weakest user:

With:

$$
D_{I}=\min \left(x_{\text {Intf }}-x_{\text {weakest User }}\right)
$$

- $D_{I}$, corresponding to the smallest distance

- $x_{\text {Intf }}$, corresponds to the coordinates of the interferers

- $\quad x_{\text {weakest } U \text { ser }}$, corresponds to the coordinates of the Beam containing the weakest user

Then the closest interferer is swapped with another one of the same spot but which is farther away from the weakest user.

\section{Permutation Technique}

As a reference, a third method has been added in order to put each value into perspective. This method consist in permuting randomly all the different frames and influence the $\mathrm{C} / \mathrm{I}$ performance. Implementing this method gives a benchmark for the proposed optimization processes.

\section{Comparative Analysis}

The three techniques have been compared on the same specific transmission scenarios based on reliable antenna data provided by Eutelsat. The first results show that both techniques help to improve a very good percentage of the different transmission scenarios.

Given a sample population of 8000 transmission scenarios (large enough population for a "reasonable" calculation time), the following results have been obtained:
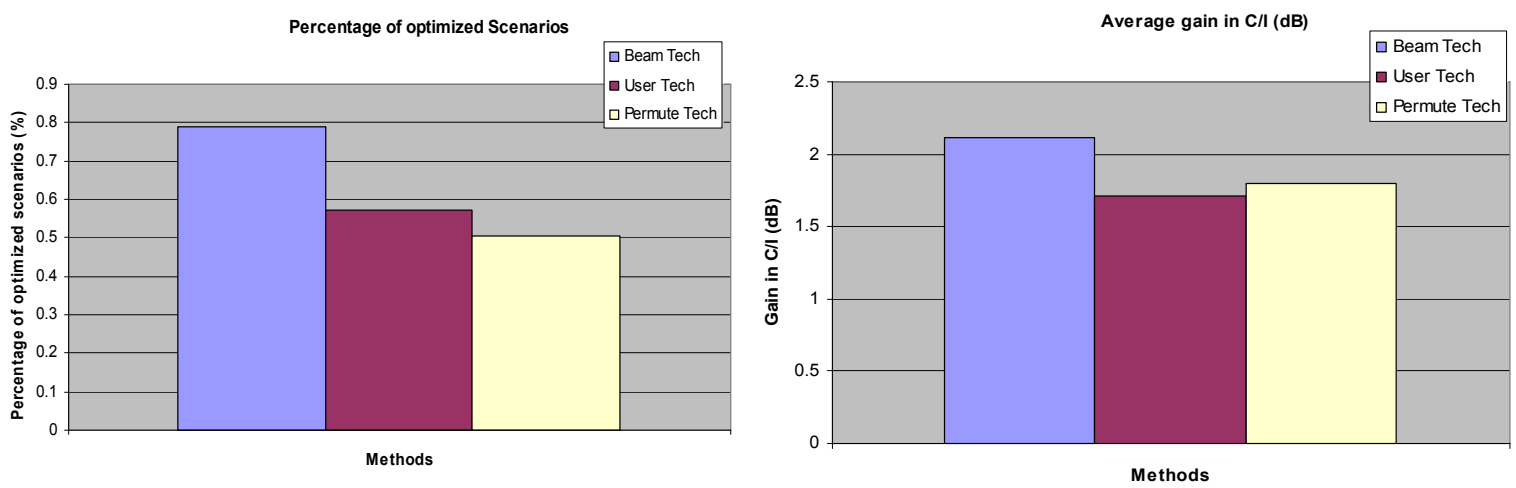

Figure 2: Optimisation percentage and average gain in $\mathrm{C} / \mathrm{I}$ for the three methods

Indeed, more than $50 \%$ of the scenarios could be improved with the user centered technique helping to gain from $0.5 \mathrm{~dB}$ to $10 \mathrm{~dB}$ of $\mathrm{C} / \mathrm{I}$ in certain constellations but on average around $1.5 \mathrm{~dB}$. This means that most of the optimizations took place in a lower range of $1 \mathrm{~dB}$ to $2 \mathrm{~dB}$.

The Beam centered technique is more efficient as it improves almost $80 \%$ of the different scenarios and with a higher average gain of around $2.1 \mathrm{~dB}$. This means that compared to the user centered method, it is also focused on a range of $1.5 \mathrm{~dB}$ to $2.5 \mathrm{~dB}$. The permutation method however shows that even if it has a slightly better average level, it has however a lower percentage than the User centered method. This implies that a certain degree of controlled optimization is necessary.

So the techniques help to achieve better C/I performances and show that they are efficient in their own ways. The average achieved gain of around $1.5 \mathrm{~dB}$ is very useful for a link budget as this could allow certain users to build up a communication in heavily interfered spot constellations. 


\section{Further Analysis}

The techniques take their full usefulness by looking at the $\mathrm{C} / \mathrm{I}$ behaviour for a given multi-beam coverage. In order to calculate the $\mathrm{C} / \mathrm{I}$ at antenna level on the Return link it is necessary to do a Monte-Carlo approach as a way to simulate the most faithfull possible the traffic behaviour. Based on this approach, it is necessary to calculate a critical amount of scenarios in order to average the correct performances explaining the 8000 sample scenario for instance.

However, as seen previously, the $\mathrm{C} / \mathrm{I}$ is heavily position dependent. Based on the constellation of users at a given moment, the performances can be very different from one moment to the other. Designing a satellite for the worst case which is quite rare (distance between a studied point and its interferers is the smallest possible) implies overdimensioning the payload in order to plan for every possibility. The consequence of such choice is more mass, power and thermal constraints on the general architecture.

The following figures show for a given antenna structure and a given studied spot (spot 13), that it is possible to observe at two different traffic moments, that the interference environment can be very different.

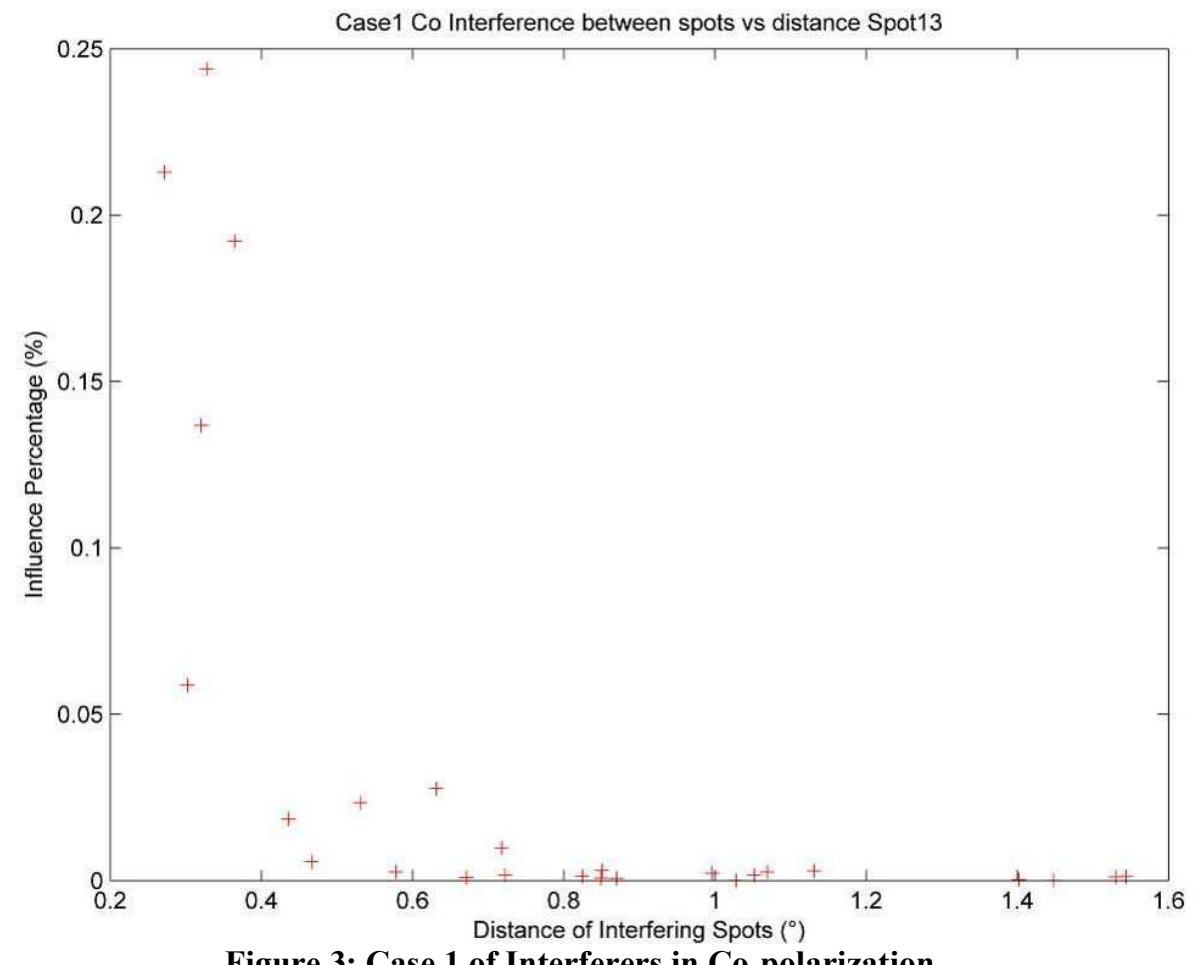

Figure 3: Case 1 of Interferers in Co-polarization 


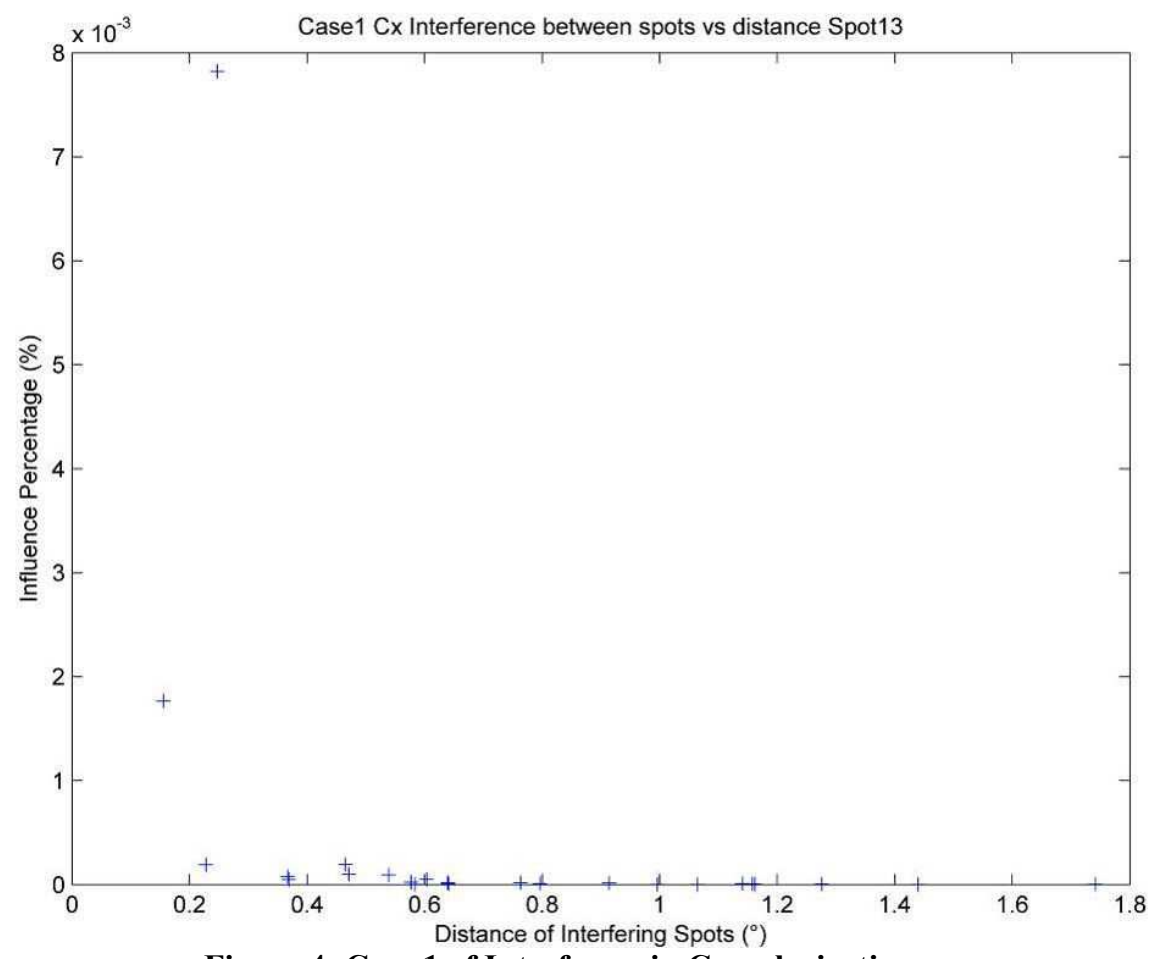

Figure 4: Case 1 of Interferers in $\mathrm{Cx}$-polarization

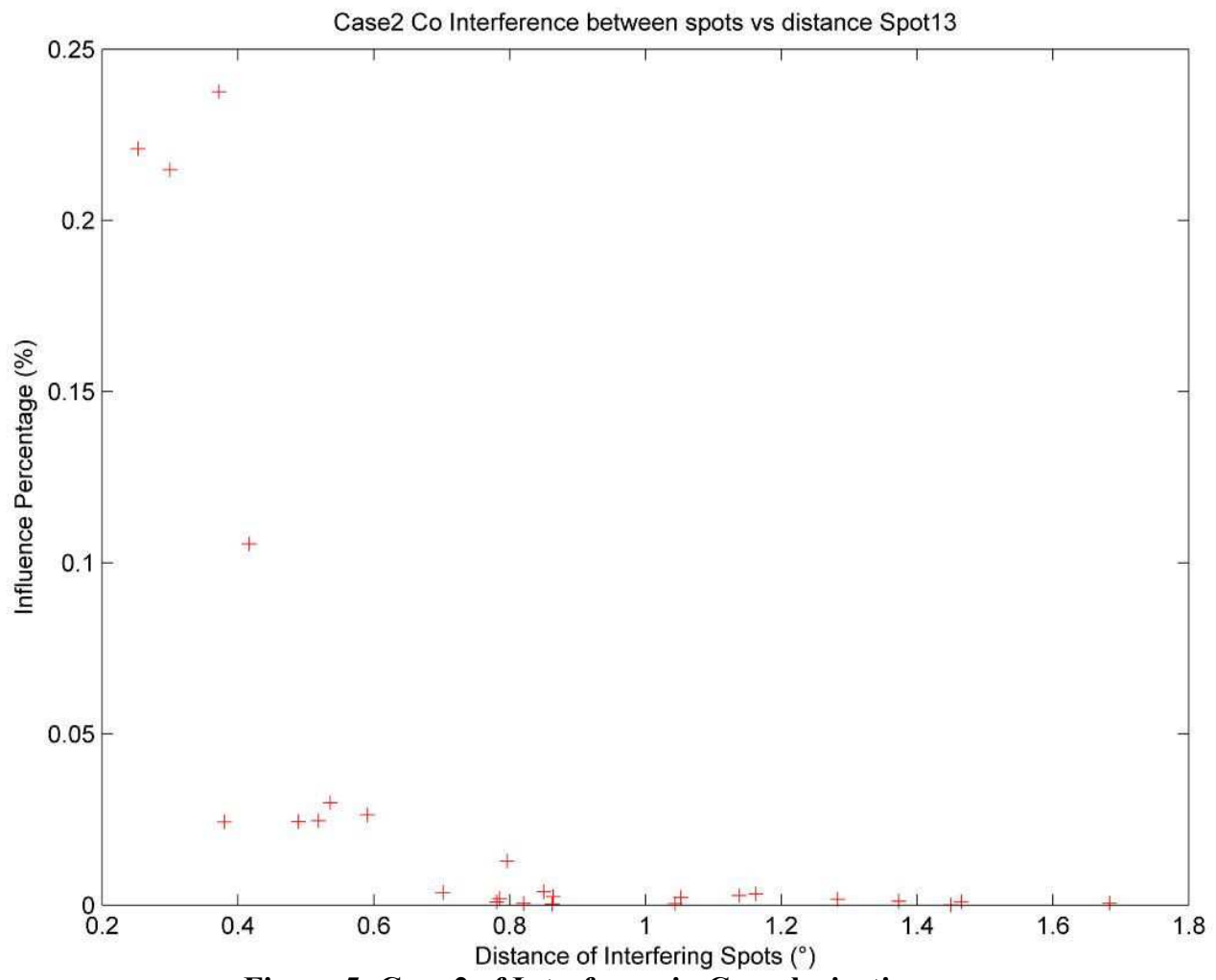

Figure 5: Case 2 of Interferers in Co-polarization 


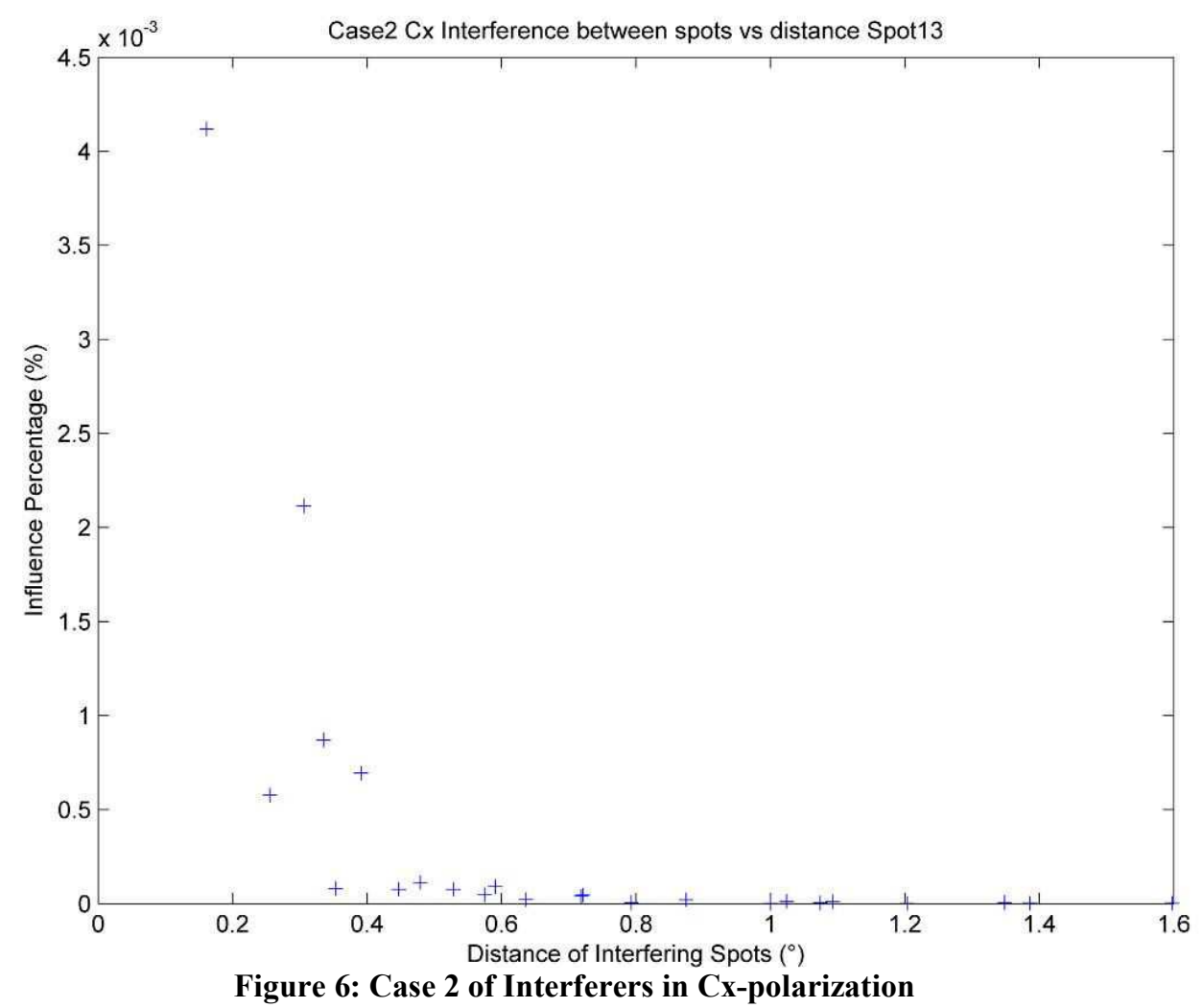

Indeed, it is clearly shown that due to the dynamic effect of the traffic the constraints vary as such. Consequently, optimizing the FTDMA frame structure based on the C/I helps to avoid overdimensioning the satellite itself and to take into account variable transmission scenarios without having to do major design revisions.

\section{Further Work}

These methods have been tested on real satellite antenna performances and achieved significant performance enhancements. Based on those first results, it is interesting to develop the two techniques and include an iterative approach for different multi beam constellations. This will help to achieve a better understanding of the methods and allow to design better coverages.

\section{Conclusion}

The first results show that those techniques can improve at a high rate the satellite communication on the return link for a broadband application. Indeed, those techniques achieve better performances in terms of C/I helping to avoid this parameter to become a bottleneck.

The technical complexity of implementation shouldn't be a show stopper as the general coding and modulation techniques are becoming more and more sophisticated and evolved giving more space for complex optimization methods.

\section{References}

Keynote Speeches

${ }^{1}$ Beretta, Keynote Speech for the 16th Ka Band Confernce, 2010

Books

${ }^{5}$ Maral and M. Bousquet, Satellite Communications Systems 3rd Edition, 2002, Wiley and sons

${ }^{6}$ Walter L. Morgan and Gary D. Gordon, Communications Satellite Handbooks, 1989, Wiley Interscience

8

American Institute of Aeronautics and Astronautics 
Articles

${ }^{2}$ Sara Alouf, Eltan Altman, Jérôme Galtier, Jean-François Lalande and Corinne Touati, Quasi-Optimal bandwidth allocation for multi-spot MFTDMA satellites, INFOCOM 2005. 24th Annual Joint Conference of the IEEE Computer and Communications Societies. Proceedings IEEE, 2005

${ }^{3}$ Sara Alouf, Eltan Altman, Jérôme Galtier, Jean-François Lalande and Corinne Touati, Un algorithme d'allocation de bande passante satellitaire, INFOCOM 2005. 24th Annual Joint Conference of the IEEE Computer and Communications Societies. Proceedings IEEE, 2004

${ }^{4}$ Wen Liu, Haixiang Shi and Lipo Wang, Minimizing Interference in Satellite Communications Using Chaotic Neural Networks, Natural Computation, 2007. ICNC 2007. Third International Conference, 2007

${ }^{7}$ P. Balling, Spacecraft Multi-Beam and Contoured-Beam Antennas, AGARD lecture, Microwave Antennas for Avionics, 1987 